\title{
PEDAL FORCE ASYMMETRIES AND PERFORMANCE DURING A 20-KM CYCLING TIME TRIAL
}

\author{
Rodrigo R. Bini' ${ }^{1}$, Tiago C. Jacques ${ }^{2}$, Carlos H. Sperb² ${ }^{2}$ Fábio J. Lanferdini², and Marco A. Vaz \\ ${ }^{1}$ Escola de Educação Física do Exército, Centro de Capacitação Física do Exército, \\ Rio de Janeiro, Brazil \\ ${ }^{2}$ Laboratório de Pesquisa do Exercício, Universidade Federal do Rio Grande do Sul, \\ Porto Alegre, Brazil
}

Original scientific paper

UDC: 796.61:796.012.11

\begin{abstract}
:
It is unclear if applying larger or more symmetrical pedal forces leads to better performance in cycling. The aims of this study were to assess the relationship between pedal force production and performance in a cycling time trial and to evaluate the relationship between asymmetries in pedal force production and performance. Fifteen competitive cyclists/triathletes performed a $20 \mathrm{~km}$ cycling time trial on a cycle trainer while bilateral forces applied to the pedals were recorded along with total time. Total forces applied to the pedals were computed and converted into dominant and non-dominant forces using a leg preference inventory. Pedal force asymmetries ranged from $43 \%$ (in favour of the dominant limb) to $34 \%$ (in favour of the non-dominant limb). The relationship between total pedal force (averaged from both pedals) and performance time was small $(\mathrm{r}=-.32$, effect $\operatorname{size}=.66)$ as well as the association between the asymmetry indices and performance time $(\mathrm{r}=.01$, effect size $=.06)$. In conclusion, applying large forces on the pedals and balancing pedal force application between the dominant and non-dominant limbs did not lead to better performance in this cycling time trial.
\end{abstract}

Key words: left/right asymmetry, Waterloo Footedness Questionnaire, pedalling technique, power output, pedalling cadence

\section{Introduction}

Studies have observed that a range from $40-60 \%$ of the force applied to the pedal is converted into crank torque (Bini, Hume, \& Kilding, 2014; Mornieux, Stapelfeldt, Gollhofer, \& Belli, 2008). Indeed, by converting a larger portion of pedal forces into crank torque, cyclists could enhance power output due to the need for lower force application on the pedals per pedal stroke (Bohm, Siebert, \& Walsh, 2008). In contrast, Lanferdini et al. (2014) have shown that cyclists with a larger power output at their anaerobic threshold rely on applying larger pedal forces, but do not show improved pedalling technique. This finding is in agreement with the previous report from Coyle et al. (1991) showing that large force production at the power stroke is indeed critical to time trial cycling performance. However, none of these studies assessed the relationship between pedal force application and performance of cyclists during time trials. This information is important given that, during a cycling time trial, pacing should be optimized by tuning muscle activations and force production in order to postpone fatigue (Bini, Carpes, Diefenthaeler, Mota, \& Guimarães, 2008; Tucker, et al., 2006). Therefore, improved cycling performance during a time trial should be associated to a larger pedal force production and not to an increased focus in pulling up the pedals at the crank cycle recovery phase (Korff, Romer, Mayhew, \& Martin, 2007). However, to date, no study assessed if cyclists that apply large pedal forces present better performance than those who apply less force on the pedals.

Existing commercial power meters have recently enabled cyclists to monitor bilateral power production (e.g. Look-Polar®). This novel information on bilateral asymmetries in power production is aligned to evidence that cyclists present an imbalance in bilateral force production from $5-20 \%$ (Carpes, Mota, \& Faria, 2010). More recent data indicated that asymmetries could be larger in uninjured cyclists (i.e. $>60 \%$ ) than the referred range of 5-20\% (Bertucci, Arfaoui, \& Polidori, 2012; Bini \& Hume, 2014) which could anticipate a risk factor for overuse injuries via an overload of one leg compared 
to the contralateral leg. In this regard, it is unclear if cyclists with larger pedal force asymmetries could be limited in terms of performance compared to the cyclists whose pedal force production is more symmetrical. Moreover, the potential link between reduced asymmetries and better performance in cycling is warranted by the cycling community, which has discussed this issue at the Wattage Google Groups forum (Wattage, 2015) in order to decide whether asymmetries should be reduced by training to improve cycling performance. To date, one study assessing the link between bilateral asymmetries in cycling and performance was limited to the assessment of only six cyclists while measuring peak crank torque (incomplete range of force production) using an unreliable instrument (Carpes, Rossato, Faria, \& Mota, 2007). The second study (Bini \& Hume, 2015) was limited to very short duration time trials (i.e. $4 \mathrm{~km}$ ) which does not provide information on longer duration tests. Therefore, there is a need to assess if pedalling, during longer duration trials, could enable cyclists to largely deviate from pedal force production symmetry, which could be observed due to the lower exercise intensity (Carpes, et al., 2007) and larger possibility to change muscle recruitment strategies.

In order to address the aforementioned questions, the goals of this study were to: 1) assess the relationship between pedal force production and performance in a $20 \mathrm{~km}$ cycling time trial and; 2) evaluate the relationship between asymmetries in pedal force production and performance in a cycling time trial. Our first hypothesis was that cyclists with better performance would produce larger pedal force because performance in cycling is related to larger pedal force application (see Lanferdini et al., 2014, for details). The second hypothesis was that increased pedal force production asymmetries would be associated with better performance because recent evidence showed that pedalling at larger loads leads to increased asymmetries (see Bini \& Hume, 2014, for details).

\section{Methods}

\section{Participants}

Fifteen athletes with competitive experience in cycling (eleven, including eight males and three females) and triathlon (four, including three males and one female) with (mean \pm SD) $37 \pm 12$ years of age, $71 \pm 13 \mathrm{~kg}$ of body mass and $177 \pm 11 \mathrm{~cm}$ of standing body height participated in the study. At the time of the evaluation sessions, cyclists/triathletes covered $331 \pm 80 \mathrm{~km} /$ week of cycling training in $5 \pm 1$ sessions per week. Although their annual training volume was greater than $17,000 \mathrm{~km}$, we ranked them as club riders (following descriptions from Ansley \& Cangley, 2009) because their projected performance at the $16 \mathrm{~km}$ under the $20 \mathrm{~km}$ time trial test was greater than 22 minutes (see later details). Cyclists/ triathletes did not report any injury or pain during racing or training over the last six months.

Before the start of the evaluation session, all procedures were presented to the participants who gave written informed consent to participate in the study which was approved by the Ethics Committee of Human Research where the study was conducted (CAEE: 09757612.1.0000.5347).

\section{Data collection}

Cyclists/triathletes completed the Waterloo Footedness Questionnaire to allow the determination of lower limb dominance (Elias, Bryden, \& Bulman-Fleming, 1998). Briefly, the inventory involves twelve questions linking lower limb preference to tasks usually performed in daily activities (e.g. If you had to hop on one foot, which foot would you use?). Cyclists/triathletes were then asked to rate their preference for right or left leg use in these tasks.

Before testing, pressure of each bicycle rear wheel was calibrated according to manufacturer's instructions ( $100 \mathrm{psi})$. Cyclists/triathletes warmed up for 10 minutes at self-selected workload and pedalling cadence ascertaining for moderate to low subjective effort. Laboratory temperature (26-28 $)$ and humidity $(\sim 50 \%)$ were controlled throughout the testing period to minimize temperature effects on bicycle tire pressure and power output measurements (Davison, Corbett, \& Ansley, 2009).

Cyclists/triathletes used their own bicycles attached to a cycle trainer (Cateye CS1000, Cateye Co., Osaka, Japan). The cycle trainer has a magnetic braking system that provides resistance proportional to the rear wheel speed (therefore, sensitive to gear ratio and pedalling cadence). After the warmup, cyclists/triathletes performed a $20 \mathrm{~km}$ cycling time trial on the cycle trainer (Sporer \& McKenzie, 2007; Zavorsky, et al., 2007). During the time trial, they were instructed to complete the $20 \mathrm{~km}$ time trial as fast as possible using self-selected strategies for gear-ratio and pedalling cadence. Elapsed time and power output were manually recorded at $5,10,15$ and $19 \mathrm{~km}$ from the cycle trainer head unit while the force applied to the pedals was recorded using a pair of strain gauge instrumented pedals (Candotti, et al., 2007) and a reed-switch attached to the bicycle frame detected crank position. The pedal force system enabled normal and anterior-posterior force measurements using strain gauges with cyclists/triathletes using cycling shoes with Look ${ }^{\circledR}$ Delta cleats. As described elsewhere (Bini \& Hume, 2013), instrumented pedals were calibrated with static loads and presented biological (due to within subjects variability) and technical reliability (due to equipment errors of measurement) of 5\% for peak pedal forces during incremented load cycling tests. 
Pedal force data passed through an amplifier (MSC-A1, Entran-MSC6, UK) and, along with reed switch signals, were recorded using an analogue-to-digital board (USB-1608G, Measurement Computing Inc, Norton, USA) at $2.4 \mathrm{kHz}$ per channel using a custom-made script in Matlab (Mathworks Inc, Natick, USA). Analogue data (force and reed switch trigger) were acquired for 30 seconds at the aforementioned distances of the $20 \mathrm{~km}$ trial. Total time was then recorded when cyclists/triathletes covered the $20-\mathrm{km}$.

\section{Data analyses}

A reed switch attached to the bicycle frame detected the position of the crank in relation to the pedal revolution and enabled to separate pedal force data into every crank revolution. Normal and anterior-posterior force signals were smoothed using a zero lag Butterworth low-pass digital filter with a cut-off frequency of $10 \mathrm{~Hz}$. Resultant (total) force applied on the sagittal plane of the pedal surface was calculated as a vector sum of normal and anterior-posterior pedal forces for a full crank cycle. Peak total pedal force was averaged for each pedal of each cyclist/triathlete across five crank revolutions for each section (i.e. 5, 10, 15 and $19 \mathrm{~km}$ ) of the $20 \mathrm{~km}$ time trial test. We used peak pedal force because this variable has better reliability than pedalling technique measures (e.g. index of effectiveness; Bini \& Hume, 2013) and because it is strongly related to power output. Average pedalling cadence was computed from timing of the reed switch sensor (Bini, et al., 2008). All force data processing was conducted using a custommade script in Matlab (Mathworks Inc, Natick, USA). After that, peak total pedal force was averaged for the entire trial, for each cyclist/triathlete, for further analyses. The asymmetry index (AI\%) was calculated as outlined by Robinson et al. (1987) for average total pedal force from the entire trial, defining positive asymmetry whenever the dominant limb (D) presented larger force measures than the non-dominant limb (ND):

$$
A 1 \%=\left[\frac{D-N D}{(D+N D) / 2}\right] \times 100
$$

\section{Statistical analyses}

Right and left measures of total pedal force were converted into measures for dominant and non-dominant limbs using results from the Waterloo Footedness Questionnaire. Briefly, responses for the twelve questions in the inventory were converted into percentages of preference. Dominance was then defined whenever there was more than $60 \%$ of preference for a given limb. After that, Student's $t$-tests were conducted (assuming heteroscedasticity in data distribution) for the comparison of dominant to non-dominant total pedal forces, in addition to Cohen's effect sizes to measure the magnitude of changes (Hopkins, Marshall, Batterham, \& Hanin, 2009). Significant differences were assumed when $\mathrm{p}<.05$ and $\mathrm{ES}>0.8$, to ascertain non-overlap in mean scores greater than 47\% (Cohen, 1988).

In order to assess the relationship between performance time (i.e. total time to cover $20 \mathrm{~km}$, in seconds) and total pedal forces, dominant and non-dominant pedal force data were averaged. In addition, the relationship between absolute values of pedal force asymmetry indices (non-negative results) and performance time was assessed using Pearson correlation coefficients, which were ranked following methods from Dancey and Reidy (2004), where $r=1.0$ indicates perfect association, $r$ between .7 and .99 indicates strong association, $r$ between .4 and .69 indicates moderate association and $r$ smaller than .39 indicates small to no association. Effect sizes for correlation coefficients and $p$-values were computed, assuming a significant relationship when $\mathrm{p}<.05$. All statistical analyses were conducted using custom-made spreadsheets (Excel, Microsoft, Inc., USA).

\section{Results}

One cyclist and one triathlete reported left leg preference (13\%) at the Waterloo Footedness Questionnaire, while the others presented right leg preference. Cyclists/triathletes covered the $20 \mathrm{~km}$ time trial in $1,802 \pm 219 \mathrm{~s}(30 \pm 3.7$ minutes $)$ with average power output recorded from the cycle trainer of $294 \pm 72 \mathrm{~W}$. Mean results for total pedal force, power output and pedalling cadence are depicted in Figure 1.

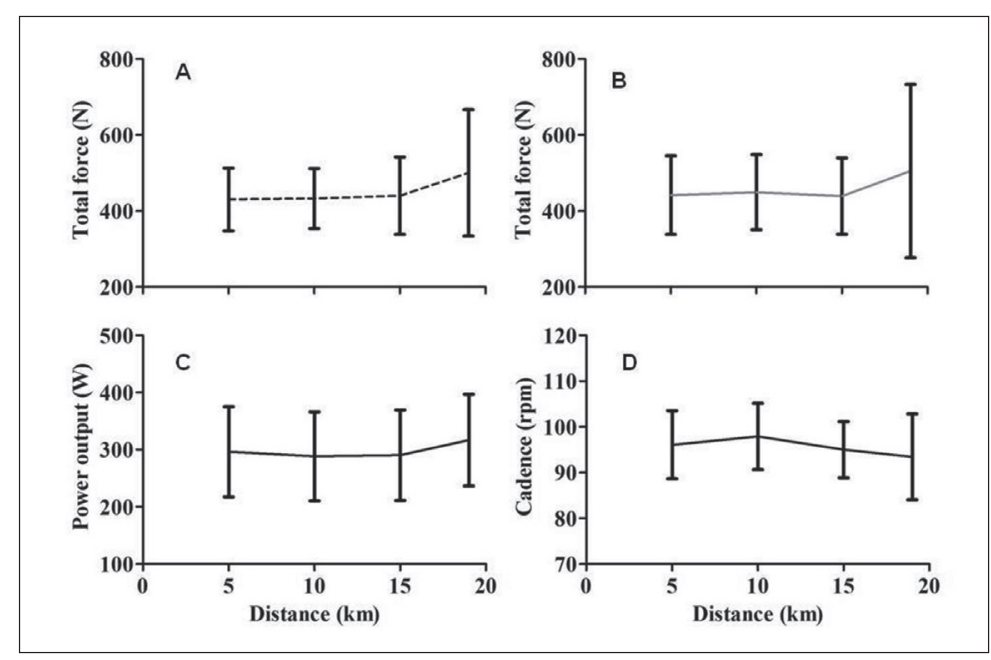

Figure 1. Grouped data (mean $\pm S D$ ) for total pedal force for the dominant (A) and non-dominant limbs (B), power output (C) and pedalling cadence (D) for the 15 cyclists/triathletes. 


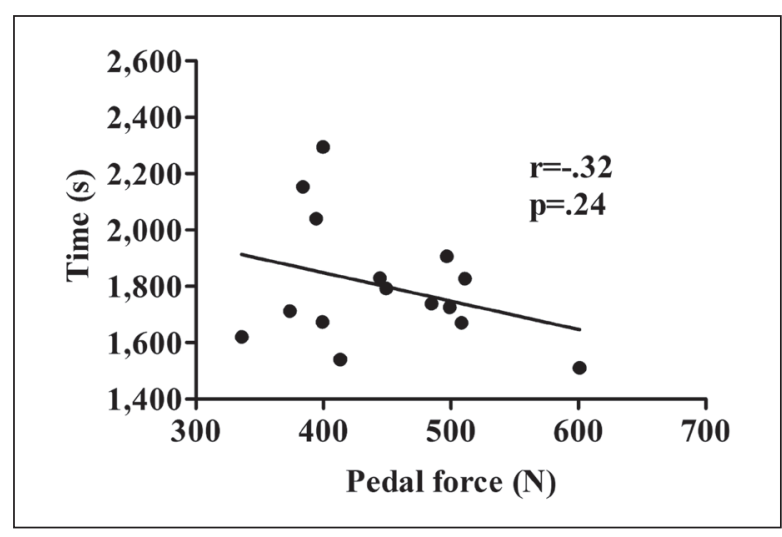

Figure 2. Relationship between total pedal force (average from dominant and non-dominant limbs) and performance time for the 15 cyclists/triathletes.

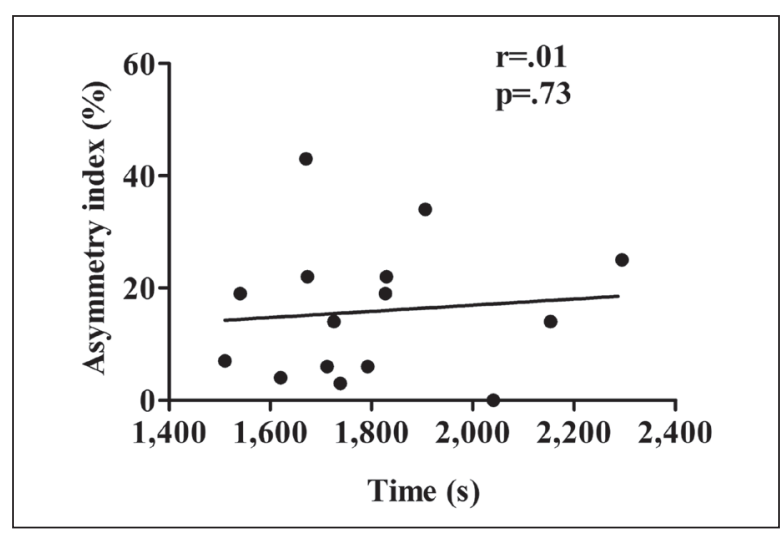

Figure 3. Relationship between absolute values of asymmetry indices (average from a $20 \mathrm{~km}$ time trial for dominant and non-dominant limbs) and performance time for the 15 cyclists/ triathletes.

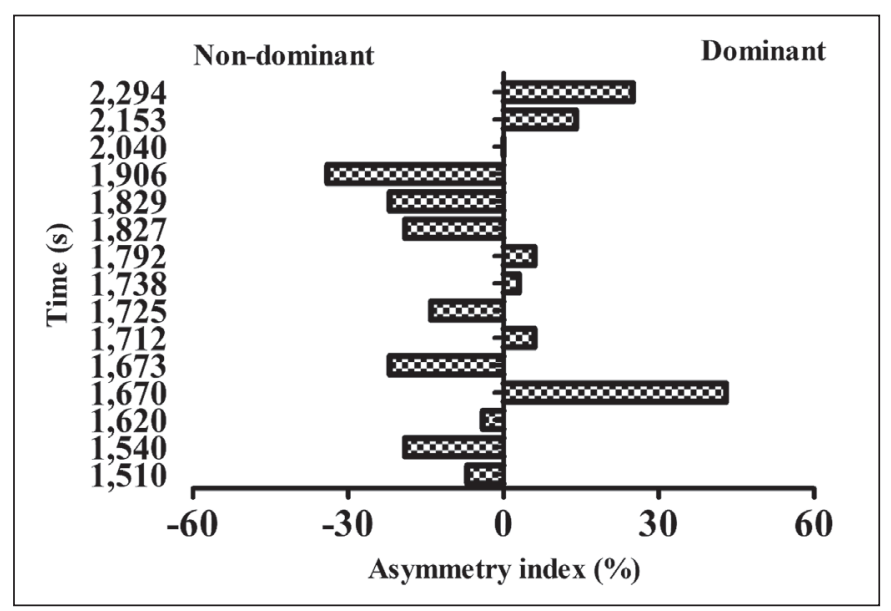

Figure 4. Individual asymmetry indices for total pedal force for the 15 cyclists/triathletes taken as averages from the $20 \mathrm{~km}$ time trial.

Peak total pedal force from the dominant limb at the $20 \mathrm{~km}$ time trial was $418 \pm 64 \mathrm{~N}$ whilst the non-dominant limb recorded $475 \pm 92 \mathrm{~N}$, which were not significantly different ( $\mathrm{p}=.06$ and $\mathrm{ES}=0.73$ ). Asymmetry index ranged from $43 \%$ (in favour of the dominant limb) to $34 \%$ (in favour of the nondominant limb) with averages of $-3 \pm 20 \%$.

The relationship between total pedal force (averaged from both pedals) and performance time was small $(r=-.32, p=.24, E S=0.66$ - see Figure 2) whilst the association between absolute asymmetry indices and performance time was also small $(\mathrm{r}=.01$, $p=.73, E S=0.06-$ see Figures 3 and 4).

Individual asymmetry indices varied largely when associated to performance time for the fifteen cyclists/triathletes, suggesting that large asymmetries are not accompanied by reduced performance time (see Figure 4).

\section{Discussion and conclusions}

This study assessed the relationship between pedal forces and performance in a $20 \mathrm{~km}$ cycling time trial along with the relationship between bilat- eral asymmetries in pedal forces and performance of cyclists/triathletes. The main findings were that larger pedal force application did not lead to better performance for the evaluated cyclists/triathletes and bilateral asymmetries were not associated to performance. These findings are novel because recent studies observed that greater total pedal forces were somewhat linked to larger power output at the anaerobic threshold (Lanferdini, et al., 2014). In parallel, only one study observed a weak link between large total pedal force production and better performance, and a weak link between asymmetries in pedal force and short duration time trial (i.e. $4 \mathrm{~km}$ ) cycling performance (Bini \& Hume, 2015). These results were confirmed by our data assessing a longer duration trial in the present study. The main difference from the current study in relation to the article by Bini and Hume (2015) is that we assessed a longer duration trial. This is critical because, during a longer cycling training, athletes are challenged to optimize their pacing strategies in order to maximize performance. Indeed, during a $4 \mathrm{~km}$ time trial (as performed in the research by Bini \& Hume, 2015) cyclists pedalled very close to their $\mathrm{VO}_{2 \max }$, which limits any room for selecting different muscle strategies, enforcing maximal pedal force application. On the other hand, during a $20 \mathrm{~km}$ time trial, we could observe that cyclists were capable to increase pedal forces close to the end of the trial ("end spurt" - as shown previously by Tucker, et al. 2006).

To enhance performance, cyclists are commonly instructed to apply the largest possible forces at the crank cycle power stroke phase compared to circling or pulling up the pedals at the recovery phase (Korff, et al., 2007). For cyclists/triathletes assessed in the present study, the largest peak total force application on the pedals did not lead to better performance. A reason could be that pedal force should be driven properly (i.e. perpendicular to the 
crank-arm) in order to generate torque in favour of crank motion. Rossato, Bini, Carpes, Diefenthaeler, and Moro (2008) observed, via increases of 20\% in pedalling cadence, that cyclists were able to significantly enhance their pedal force effectiveness. These authors stated that cyclists can optimize pedal force effectiveness by improving force directions during the power stroke when pedalling at faster cadences. Therefore, cyclists/triathletes should focus on driving larger pedal forces as close as possible to the 3 o'clock crank position (i.e. $90^{\circ}$ of crank angle) in order to convert the greatest possible proportion of their pedal force into crank torque. An alternative could be to increase pedalling cadence in order to enhance inertial contribution to pedal forces for a given muscular contribution (Loras, Ettema, \& Leirdal, 2009).

A qualitative assessment of average power output from our cyclists/triathletes indicates that most of these athletes opted for an all out strategy towards the end of the time trial, similarly to observations from previous studies (Bini, et al., 2008; Tucker, et al., 2006). This strategy was accomplished by either an increase in pedal forces linked to a decrease in pedalling cadence or vice versa. In our study, cyclists/triathletes had full control of their gear ratio (which affects pedal forces) and pedalling cadence in order to optimize their pacing strategy. Therefore, the combination between pedal force and cadence should affect power output and performance in time trials, which may have reduced the relationship between pedal force and performance time in our study. However, in a previous study, Bini et al. (2008) did not observe a relationship between pedalling cadence and cycling performance. Therefore, it is uncertain whether cyclists/triathletes should focus on increasing the power output by pedalling at higher cadences or by changing gears for a given cadence. Individual strategies should be assessed via sprint tests (Dorel, et al., 2010) which could provide an outline of the force-velocity properties for each cyclist/triathlete.

An inverse relationship between bilateral asymmetries in pedal forces and cycling performance could have been expected assuming that towards maximal exercise effort, inter-hemispheric cortical communication should be enhanced to provide full neural drive to lower limbs (Carpes, Mota, et al., 2010). However, both increases (Bini \& Hume, 2014) and decreases (Carpes, et al., 2007) in bilateral asymmetries were observed towards maximal exercise effort. In the case of the $20 \mathrm{~km}$ time trial, exercise intensity is close to $\sim 70 \%$ of maximal aerobic power output (Sporer \& McKenzie, 2007) which does not induce maximal exercise effort. These findings indicate that asymmetries could be tuned and some cyclists/triathletes may opt for driving the pedals stronger using either their dominant or the non-dominant limb, as shown in Figure 3.

Evidence from muscle activation indicates that both the dominant and non-dominant limbs could present similar efficiencies during single leg cycling (Carpes, Diefenthaeler, et al., 2010). In contrast, larger mechanical load applied to the bones by the dominant limb during growth could lead to increases in bone enthesis (Kanchan, Mohan Kumar, Pradeep Kumar, \& Yoganarasimha, 2008) and potential changes in muscle moment-arm. Similarly, recent findings indicate that differential load applied to the dominant lower limb could determine $\sim 30 \%$ greater stiffness for the Achilles tendon (Bohm, Mersmann, Marzilger, Schroll, \& Arampatzis, 2015). Taken together, it is uncertain if larger bilateral asymmetries are detrimental to performance or if they are a natural consequence from adaptation of musculoskeletal tissues to limb preference.

The most suggested range for normative asymmetries in cycling is between $5-20 \%$ of bilateral forces differences (Carpes, Mota, et al., 2010) for uninjured cyclists and up to $400 \%$ in injured cyclists (Hunt, Sanderson, Moffet, \& Inglis, 2004; Mimmi, Pennacchi, \& Frosini, 2004). Our cyclists/triathletes presented bilateral asymmetries in peak pedal forces up to $43 \%$, which is similar to the observed in previous studies (Bertucci, et al., 2012; Bini \& Hume, 2014), but larger than in other studies (Bini, Diefenthaeler, Carpes, \& Mota, 2007; GarciaLopez, Diez-Leal, Larrazabal, \& Ogueta-Alday, 2015). However, differences in variables used to compute bilateral asymmetries in cycling (i.e. crank torque, total pedal force, crank power) should be taken into account when comparing results from different studies. More research is needed to provide, perhaps, a wider band for normative asymmetries in uninjured cyclists/triathletes, which could indicate cyclist who should follow an intervention to reduce bilateral asymmetries in order to prevent overuse injuries (Carpes, Mota, et al., 2010).

Among the limitations of the present study, pedal and lower limb kinematics could have been measured in order to compute potential asymmetries in joint kinetics, which could have helped to assess any sources of asymmetries in pedal forces.

In summary, cyclists/triathletes that opted for applying large pedal force did not perform better than their counterparts. Larger bilateral asymmetries in pedal forces were apparently not detrimental to performance because some cyclists/triathletes with large asymmetries performed better than others with reduced asymmetries. Mean asymmetries were in a wider range (up to $43 \%$ ) than normative for uninjured cyclists (5-20\%), which could impose a need to revisit the normative ranges for asymmetries in cycling. 


\section{References}

Ansley, L., \& Cangley, P. (2009). Determinants of “optimal” cadence during cycling. European Journal of Sport Science, 9(2), 61-85. doi: 10.1080/17461390802684325

Bertucci, W.M., Arfaoui, A., \& Polidori, G. (2012). Analysis of the pedaling biomechanics of master's cyclists: A preliminary study. Journal of Science and Cycling, 1(2), 42-46.

Bini, R.R., \& Hume, P.A. (2013). Between-day reliability of pedal forces for cyclists during an incremental cycling test to exhaustion. Isokinetics and Exercise Science, 21(3), 203-209. doi: 10.3233/ies-130510

Bini, R.R., \& Hume, P.A. (2014). Assessment of bilateral asymmetry in cycling using commercial instrumented crank system and instrumented pedals. International Journal of Sports Physiology and Performance, 9(5), 880-885. doi: 10.1123/ijspp.2013-0494

Bini, R.R., \& Hume, P.A. (2015). Relationship between force asymmetry and performance in cycling time trial. Journal of Sports Medicine and Physical Fitness, 55(9), 892-898.

Bini, R., Hume, P., \& Kilding, A. (2014). Saddle height effects on pedal forces, joint mechanical work and kinematics of cyclists and triathletes. European Journal of Sport Science, 14(1), 44-52. doi: 10.1080/17461391.2012.725105

Bini, R.R., Carpes, F.P., Diefenthaeler, F., Mota, C.B., \& Guimarães, A.C.S. (2008). Physiological and electromyographic responses during 40-km cycling time trial: Relationship to muscle coordination and performance. Journal of Science and Medicine in Sport, 11(4), 363-370. doi: 10.1016/j.jsams.2007.03.006

Bini, R.R., Diefenthaeler, F., Carpes, F.P., \& Mota, C.B. (2007). External work bilateral symmetry during incremental cycling exercise. Paper presented at the 25 International Symposium on Biomechanics in Sports, Ouro Preto, Brazil. Retrieved from: http:/w4.ub.uni-konstanz.de/cpa/article/view/427 on September 22, 2015.

Bohm, S., Mersmann, F., Marzilger, R., Schroll, A., \& Arampatzis, A. (2015). Asymmetry of Achilles tendon mechanical and morphological properties between both legs. Scandinavian Journal of Medicine and Science in Sports, 25(1), e124-e132. doi: 10.1111/sms.12242

Bohm, H., Siebert, S., \& Walsh, M. (2008). Effects of short-term training using SmartCranks on cycle work distribution and power output during cycling. European Journal of Applied Physiology, 103(2), 225-232. doi: 10.1007/ s00421-008-0692-z

Candotti, C.T., Ribeiro, J., Soares, D.P., de Oliveira, A.R., Loss, J.F., \& Guimarães, A.C.S. (2007). Effective force and economy of triathletes and cyclists. Sports Biomechanics, 6(1), 31-43. doi: 10.1080/14763140601058490

Carpes, F.P., Diefenthaeler, F., Bini, R.R., Stefanyshyn, D., Faria, I.E., \& Mota, C.B. (2010). Does leg preference affect muscle activation and efficiency? Journal of Electromyography and Kinesiology, 20(6), 1230-1236. doi: 10.1016/j.jelekin.2010.07.013

Carpes, F.P., Mota, C.B., \& Faria, I.E. (2010). On the bilateral asymmetry during running and cycling - A review considering leg preference. Physical Therapy in Sport, 11(4), 136-142. doi: 10.1016/j.ptsp.2010.06.005

Carpes, F.P., Rossato, M., Faria, I.E., \& Mota, C.B. (2007). Bilateral pedaling asymmetry during a simulated 40-km cycling time-trial. Journal of Sports Medicine and Physical Fitness, 47(1), 51-57.

Cohen, J. (1988). Statistical power analysis for the behavioral sciences (2 ${ }^{\text {nd }}$ ed; Vol. 1). Hillsdale, NJ: Routledge Academic Press.

Coyle, E.F., Feltner, M.E., Kautz, S.A., Hamilton, M.T., Montain, S.J., \& Baylor, A.M. (1991). Physiological and biomechanical factors associated with elite endurance cycling performance. Medicine and Science in Sports and Exercise, 23(1), 93-107.

Dancey, C.P., \& Reidy, J. (2004). Statistics without maths for psychology: Using SPSS for Windows. Hertfordshire, UK: Prentice Hall International.

Davison, R.C.R., Corbett, J., \& Ansley, L. (2009). Influence of temperature and protocol on the calibration of the Computrainer electromagnetically-braked cycling ergometer. International SportMed Journal, 10(2), 66-76.

Dorel, S., Couturier, A., Lacour, J. R., Vandewalle, H., Hautier, C., \& Hug, F. (2010). Force-velocity relationship in cycling revisited: Benefit of two-dimensional pedal forces analysis. Medicine and Science in Sports and Exercise, 42(6), 1174-1183. doi: 10.1249/MSS.0b013e3181c91f35

Elias, L.J., Bryden, M.P., \& Bulman-Fleming, M.B. (1998). Footedness is a better predictor than is handedness of emotional lateralization. Neuropsychologia, 36(1), 37-43. doi: 10.1016/S0028-3932(97)00107-3

Garcia-Lopez, J., Diez-Leal, S., Larrazabal, J., \& Ogueta-Alday, A. (2015). No bilateral asymmetry during pedalling in healthy cyclists of different performance levels. Paper presented at the 33rd International Conference on Biomechanics in Sports, Poitiers, France.

Hopkins, W.G., Marshall, S.W., Batterham, A.M., \& Hanin, J. (2009). Progressive statistics for studies in sports medicine and exercise science. Medicine and Science in Sports and Exercise, 41(1), 3-12. doi: 10.1249/ MSS.0b013e31818cb278

Hunt, M.A., Sanderson, D.J., Moffet, H., \& Inglis, J.T. (2004). Interlimb asymmetry in persons with and without an anterior cruciate ligament deficiency during stationary cycling. Archives of Physical Medicine and Rehabilitation, 85(9), 1475-1478. doi: 10.1016/j.apmr.2003.10.017

Kanchan, T., Mohan Kumar, T.S., Pradeep Kumar, G., \& Yoganarasimha, K. (2008). Skeletal asymmetry. Journal of Forensic and Legal Medicine, 15(3), 177-179. doi: 10.1016/j.jflm.2007.05.009 
Korff, T., Romer, L.M., Mayhew, I., \& Martin, J.C. (2007). Effect of pedaling technique on mechanical effectiveness and efficiency in cyclists. Medicine and Science in Sports and Exercise, 39(6), 991-995. doi: 10.1249/ mss.0b013e318043a235

Lanferdini, F.J., Bini, R.R., Cunha, G., Lopes, A.L., Castro, F.A., \& Oliveira, A.R. (2014). Relationship between physiological and biomechanical variables with aerobic power output in cycling. Journal of Science and Cycling, $3(1), 9-15$.

Loras, H., Ettema, G., \& Leirdal, S. (2009). The muscle force component in pedaling retains constant direction across pedaling rates. Journal of Applied Biomechanics, 25(1), 85-92.

Mimmi, G., Pennacchi, P., \& Frosini, L. (2004). Biomechanical analysis of pedalling for rehabilitation purposes: Experimental results on two pathological subjects and comparison with non-pathological findings. Computer Methods in Biomechanics and Biomedical Engineering, 7(6), 339-345. doi: 10.1080/10255840412331332951

Mornieux, G., Stapelfeldt, B., Gollhofer, A., \& Belli, A. (2008). Effects of pedal type and pull-up action during cycling. International Journal of Sports Medicine, 29(10), 817-822. doi: 10.1055/s-2008-1038374

Robinson, R.O., Herzog, W., \& Nigg, B.M. (1987). Use of force platform variables to quantify the effects of chiropractic manipulation on gait symmetry. Journal of Manipulative and Physiological Therapeutics, 10(4), 172-176.

Rossato, M., Bini, R.R., Carpes, F.P., Diefenthaeler, F., \& Moro, A.R.P. (2008). Cadence and workload effects on pedaling technique of well-trained cyclists. International Journal of Sports Medicine, 29(9), 746-752. doi: $10.1055 / \mathrm{s}-2008-1038375$

Sporer, B.C., \& McKenzie, D.C. (2007). Reproducibility of a laboratory based 20-km time trial evaluation in competitive cyclists using the velotron pro ergometer. International Journal of Sports Medicine, 28(11), 940-944. doi: $10.1055 / \mathrm{s}-2007-964977$

Tucker, R., Bester, A., Lambert, E.V., Noakes, T.D., Vaughan, C.L., \& St. Clair Gibson, A. (2006). Non-random fluctuations in power output during self-paced exercise. British Journal of Sports Medicine, 40(11), 912-917. doi: 10.1136/bjsm.2006.026435

Wattage. (2015). Left/right balance - What's acceptable and how can I improve? Retrieved from https://groups.google. com/d/topic/wattage/VWU5MTPKGCw/discussion on March 8, 2015.

Zavorsky, G.S., Murias, J.M., Gow, J., Kim, D.J., Poulin-Harnois, C., \& Kubow, S. (2007). Laboratory 20-km cycle time trial reproducibility. International Journal of Sports Medicine, 28(9), 743-748. doi: 10.1055/s-2007-964969

\author{
Submitted: September 22, 2015 \\ Accepted: March 10, 2016 \\ Correspondence to: \\ Rodrigo Bini, Ph.D., Lecturer \\ La Trobe Rural Health School \\ College of Science, Health and Engineering \\ La Trobe University \\ Flora Hill Campus, Bendigo, Victoria, 3550, \\ Australia \\ E-mail: r.bini@latrobe.edu.au
}

\title{
Acknowledgements
}

The authors acknowledge CNPq for funding (grants code 500865/2012-4) and the University for supporting this research. Thanks are given to participants who volunteered for this study.

\section{Conflict of interest statement}

The authors declare no existing conflict of interest for the contents of the article. 